CERN-EP/2004-073

28 December 2004

\title{
ISOL BEAMS OF NEUTRON-RICH OXYGEN ISOTOPES
}

U. Köster ${ }^{1)}$, O. Arndt' ${ }^{2)}$, U. Bergmann ${ }^{1)}$, R. Catherall ${ }^{1)}$, J. Cederkäl1 ${ }^{1)}$, I. Dillmann' ${ }^{2}$, M. Dubois ${ }^{3)}$, F. Durantel ${ }^{3)}$, L. Fraile ${ }^{1)}$, S. Franchoo ${ }^{1)}$, G. Gaubert ${ }^{3)}$, L. Gaudefroy ${ }^{4)}$, O. Hallmann ${ }^{2}$, C. Huet-Equilbec ${ }^{3)}$, B. Jacquot ${ }^{3)}$, P. Jardin ${ }^{3)}$, K.L. Kratz ${ }^{2)}$, N. Lecesne ${ }^{3)}$, R. Leroy ${ }^{3)}$, A. Lopez ${ }^{3)}$, L. Maunoury ${ }^{3)}$, J.Y. Pacquet ${ }^{3)}$, B. Pfeiffer ${ }^{2)}$, M.G. Saint-Laurent ${ }^{3)}$, C. Stodel ${ }^{3)}$, A.C.C. Villari ${ }^{\left.3)^{5}\right)}$ and L. Weissman ${ }^{6)}$

\begin{abstract}
ISOL beams of ${ }^{19-22} \mathrm{O}$ were produced at ISOLDE and GANIL. At ISOLDE the neutron-rich oxygen isotopes are produced by $1.4 \mathrm{GeV}$ proton-induced reactions in a $\mathrm{UC}_{x}$ /graphite target. The target is connected via a water-cooled transfer line (to retain all non-volatile isobars) to an ISOLDE type FEBIAD ion source where the released $\mathrm{CO}$ is dominantly ionized as $\mathrm{CO}^{+} .{ }^{19-22} \mathrm{O}$ beams were also produced at SPIRAL (GANIL). A $77.5 \mathrm{MeV} /$ nucleon ${ }^{36} \mathrm{~S}$ beam was fragmented in a thick graphite target, coupled by a cold transfer tube to an ECR ion source which ionizes the released $\mathrm{CO}$ dominantly as $\mathrm{O}^{+}$and $\mathrm{CO}^{+}$.
\end{abstract}

(Submitted to European Physical Journal A)

1) CERN, ISOLDE, 1211 Genève 23, Switzerland

2) Institut für Kernchemie, Universität Mainz, 55128 Mainz, Germany

3) GANIL, IN2P3-CNRS/DSM-CEA, B.P. 55027, 14076 Caen Cedex 5, France

4) Institut de Physique Nucléaire d'Orsay, 91406 Orsay Cedex, France

5) Physics Division, Argonne National Laboratory, IL-60439, USA

6) NSCL, Michigan State University, East Lansing, MI-48824, USA 
Radioactive ion beams are mainly produced by two different methods: either by in-flight separation of fast reaction products created in projectile fragmentation, fusionevaporation or fission reactions or by the isotope separation on-line (ISOL) method. In the latter the reaction products are first stopped in a thick target. From the target, typically being heated to high temperatures, the reaction products can diffuse and effuse out towards an attached ion source where they are ionized, extracted, accelerated to few ten $\mathrm{keV}$ and mass-separated. ISOL beams have normally the advantage of higher beam intensity (since thicker targets can be used) and higher beam quality (smaller emittance, small energy spread), but not all elements are easily released from the thick target, thus leading to significant decay losses for short-lived isotopes.

Oxygen is a more "difficult" element for ISOL facilities due to its high chemical reactivity. Atomic oxygen would easily react at each surface collision in the target and ion source unit, thus getting retained for too long time. To favor a rapid release, the oxygen radicals have to be bound as soon as possible in a volatile, but less reactive molecule. A suitable molecule is $\mathrm{CO}$ which is readily formed by reaction of oxygen radicals with hot carbon. Thus targets made from pure graphite or graphite mixed with other materials are expected to be favorable for the fast release of radioactive oxygen isotopes.

Beams of the neutron-deficient oxygen isotopes ${ }^{14,15} \mathrm{O}$ have been produced at several ISOL facilities, see e.g. [1] for a review. In the present article we discuss recent results and future prospects of the production of ISOL beams of neutron-rich oxygen isotopes at ISOLDE and SPIRAL.

\section{Neutron-rich oxygen beams at ISOLDE}

At ISOLDE neutron-rich oxygen isotopes are produced by high-energy protoninduced reactions of heavy target materials. Previously beams of ${ }^{19-22} \mathrm{O}^{12} \mathrm{C}^{+}[2]$ had been obtained from a mixed $26.8 \mathrm{~g} / \mathrm{cm}^{2} \mathrm{Pt} /$ graphite powder target bombarded with $0.6 \mathrm{GeV}$ protons and connected to a special ISOLDE-type FEBIAD ion source with tungsten cathode and graphite plasma chamber. Recently such beams were also produced by $1.4 \mathrm{GeV}$ proton bombardment of a $44 \mathrm{~g} / \mathrm{cm}^{2}$ standard ISOLDE $\mathrm{UC}_{x} /$ graphite target. The $2000^{\circ} \mathrm{C}$ hot target is connected via a water-cooled transfer line (to retain all non-volatile isobars) to an ISOLDE MK7-type FEBIAD ion source [3] where the released CO is dominantly ionized as $\mathrm{CO}^{+}$. Fig. 1 shows the relative population of atomic oxygen ions and molecular sidebands as measured with stable and radioactive oxygen tracers. Since abundant amounts of stable $\mathrm{CO}$ are already released from the hot target and ion source unit it is difficult to measure the absolute ion source efficiency by injecting $\mathrm{CO}$ with natural isotopic composition through a calibrated leak. Thus, the displayed absolute ionization efficiency for $\mathrm{CO}^{+}$of $3.7 \%$ was obtained by scaling measured efficiencies of the noble gases $\mathrm{He}, \mathrm{Ne}, \mathrm{Ar}, \mathrm{Kr}$ and $\mathrm{Xe}$ with the known electron impact ionization cross-sections $[4,5,6]$ and an $A^{-1 / 2}$ mass dependence which represents the transit time through the plasma chamber. However, the overall efficiency for release and ionization of radioactive oxygen isotopes is indeed much lower, due to strong getter losses at the $1900^{\circ} \mathrm{C}$ hot tantalum cathode and tantalum target enclosure. Comparing the measured ${ }^{19} \mathrm{O}^{12} \mathrm{C}^{+}$beam intensity (see Fig. 2) with the $0.29(8) \mathrm{mb}$ production cross-section measured in inverse kinematics $\left(1 \mathrm{GeV} /\right.$ nucleon ${ }^{238} \mathrm{U}$ on $\left.{ }^{1} \mathrm{H}\right)$ at GSI $[7,8]$ gives an overall efficiency of only $0.03 \%$. In fact, the cross-sections for production of light isotopes in proton-induced reactions on heavy targets rise typically by a factor two or more when increasing the proton energy from $1.0 \mathrm{GeV}$ to $1.4 \mathrm{GeV}[1]$. Hence, the real overall efficiency is probably even 
lower, indicating that $>99 \%$ of the produced ${ }^{19} \mathrm{O}$ gets trapped somewhere and is never released.

The yields from the $\mathrm{UC}_{x}$ /graphite target are $50 \%$ to $300 \%$ higher than those formerly measured with the Pt/graphite target and a short test run with a ${ }^{22} \mathrm{O}^{12} \mathrm{C}^{+}$beam gave already new nuclear structure information [9]. The only observable contamination of the ${ }^{x} \mathrm{O}^{12} \mathrm{C}^{+}$beams stems from ${ }^{x-1} \mathrm{O}^{13} \mathrm{C}^{+}$which is of the order of few percent $(1.1 \%$ abundance of ${ }^{13} \mathrm{C}$ times the yield ratio for $\left.{ }^{x-1} \mathrm{O} /{ }^{x} \mathrm{O}\right)$.

\section{Neutron-rich oxygen beams at SPIRAL}

At GANIL neutron-rich oxygen isotopes were produced by fragmenting a 77.5 $\mathrm{MeV} /$ nucleon ${ }^{36} \mathrm{~S}$ beam in the SPIRAL graphite target [10]. Oxygen is again forming CO which reaches via a cold transfer tube the NANOGAN3 ECR ion source. The latter (plasma chamber at room temperature) is optimized for the production of multiply charged ions, but delivers also singly charged atoms and ions. The ionization efficiencies of the different ion species have been measured with a ${ }^{13} \mathrm{C}^{16} \mathrm{O}$ tracer [11] and are displayed for comparison in Fig. 1. Compared to the FEBIAD ion source a much higher fraction of multiply charged oxygen ions are present as well as a considerable amount of $\mathrm{HO}^{+}$and $\mathrm{H}_{2} \mathrm{O}^{+}$. These, stemming from water vapor, are significantly suppressed by the hot plasma chamber of the FEBIAD.

The beam intensities measured in two different test runs (with $0.8 \mathrm{~kW}$ to $1.4 \mathrm{~kW}$ primary beam power) were scaled to $1.5 \mathrm{~kW}$ of primary beam power and displayed in Fig. 2. If other than singly charged atomic oxygen ions had been measured, the beam intensity was scaled to $\mathrm{O}^{+}$with the relative ionization efficiencies from Fig. 1. The shown intensities are fully consistent with those previously measured at the SHyPIE set-up [12] when taking into account the lower beam power and a roughly twice lower ionization efficiency.

\section{Conclusion and outlook}

Although the obtained beam intensities are already sufficient to perform certain experiments, there is clear room for improvement:

1. Replacing at ISOLDE the FEBIAD by a $1+$ ECR ion source without hot Ta parts and lining the entire Ta target container with graphite would strongly reduce the gettering losses and may result in a gain of up to three orders of magnitude in yield.

2. The GANIL yields of ${ }^{19,20} \mathrm{O}$ could by enhanced by about a factor five (calculated with EPAX V2.1 [13]) by replacing the primary ${ }^{36} \mathrm{~S}$ beam with a ${ }^{22} \mathrm{Ne}$ beam of the same beam power. A straightforward gain of a factor of four is expected from the THI upgrade to $6 \mathrm{~kW}$ primary beam intensity [14].

3. An extension towards ${ }^{23,24} \mathrm{O}$ is more difficult, not only due to the lower production cross-section and the very short half-life (increasing the decay losses), but mainly due to the background situation. Atomic ${ }^{23} \mathrm{O}^{+}$beams suffer from very intense ${ }^{23} \mathrm{Ne}^{+}$ background and molecular ${ }^{12} \mathrm{C}^{23} \mathrm{O}^{+}$beams have strong background from ${ }^{35} \mathrm{Ar}^{+}$and at ISOLDE moreover from the abundantly produced fission product ${ }^{140} \mathrm{Xe}^{4+}$. However, this background could be removed, by:

- A gas-filled beam manipulation device (Penning trap or RFQ cooler) which resets the multiply charged background to the $1+$ charge state, followed by a $A / q$ separation to suppress it.

- Molecular beams could be separated from atomic background by breaking the 
molecules (via stripping or charge-breeding) and subsequent second mass separation.

\section{Acknowledgements}

Supported by the EU-RTD project TARGISOL (contract HPRI-CT-2001-50033). ACCV acknowledges his partial support by the U.S. Dept. of Energy, Office of Nucl. Phys. (contract W31-109-ENG-38).

\section{References}

[1] U. Köster, Eur. Phys. J. A 15 (2002), 255.

[2] ISOLDE SC yield database, http://www.cern.ch/ISOLDE/normal/isoprodsc.html.

[3] S. Sundell, H. Ravn and the ISOLDE Collaboration, Nucl. Instr. Meth. B 70 (1992), 160.

[4] H.C. Straub, B.G. Lindsay, K.A. Smith and R.F. Stebbings, J. Chem. Phys. 105 (1996), 4015.

[5] M.A. Mangan, B.G. Lindsay and R.F. Stebbings, J. Phys. B 33 (2000), 3225.

[6] R. Rejoub, B.G. Lindsay and R.F. Stebbings, Phys. Rev. A 65 (2002), 042713.

[7] P. Armbruster et al., Phys. Rev. Lett. 93 (2004), 212701.

[8] Maria-Valentina Ricciardi, High-resolution measurements of light nuclides produced in $1 \mathrm{~A} \mathrm{GeV}{ }^{238} \mathrm{U}$-induced reactions in hydrogen and in titanium, Ph.D. thesis, Universidad de Santiago de Compostela (2005).

[9] L. Weissman et al., J. Phys. G 31 (2005) 553.

[10] J.C. Putaux et al., Nucl. Instr. Meth. B 126 (1997), 113.

[11] S. Gibouin et al., Nucl. Instr. Meth. B 204 (2003), 240.

[12] Nathalie Lecesne, Etude de la production d'ions radioactifs multichargés en ligne, Ph.D. thesis, Université de Caen (1997).

[13] K. Sümmerer and B. Blank, Phys. Rev. C 61 (2000), 034607.

[14] E. Baron et al., in 15th Int. Conf. Cyclotrons and Applications, 14-19 June 1998, Caen, France, edited by E. Baron and M. Lieuvin, pp. 385-388, IOP (1999). 


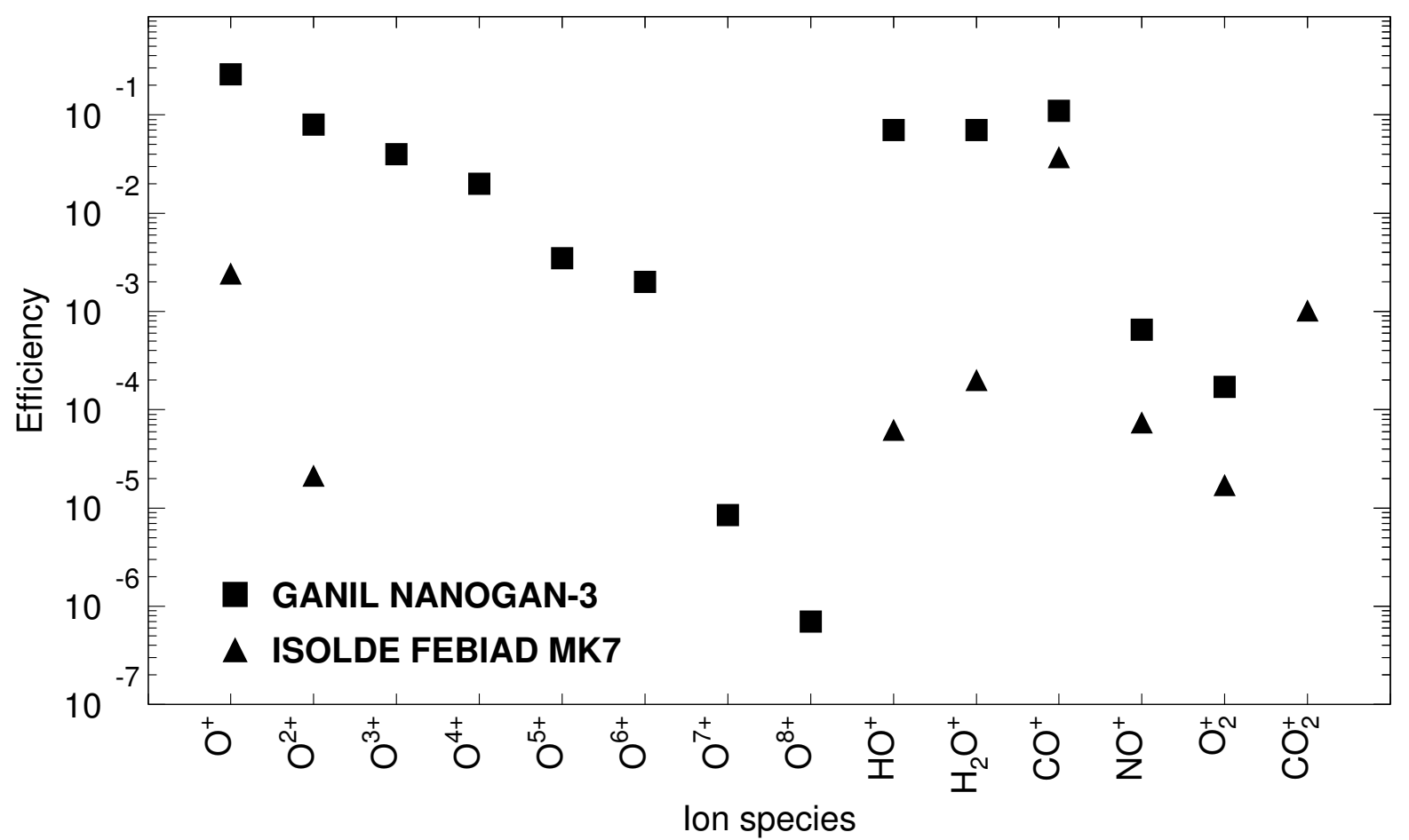

Figure 1: Measured relative and absolute ionization efficiencies for different oxygen containing ion species.

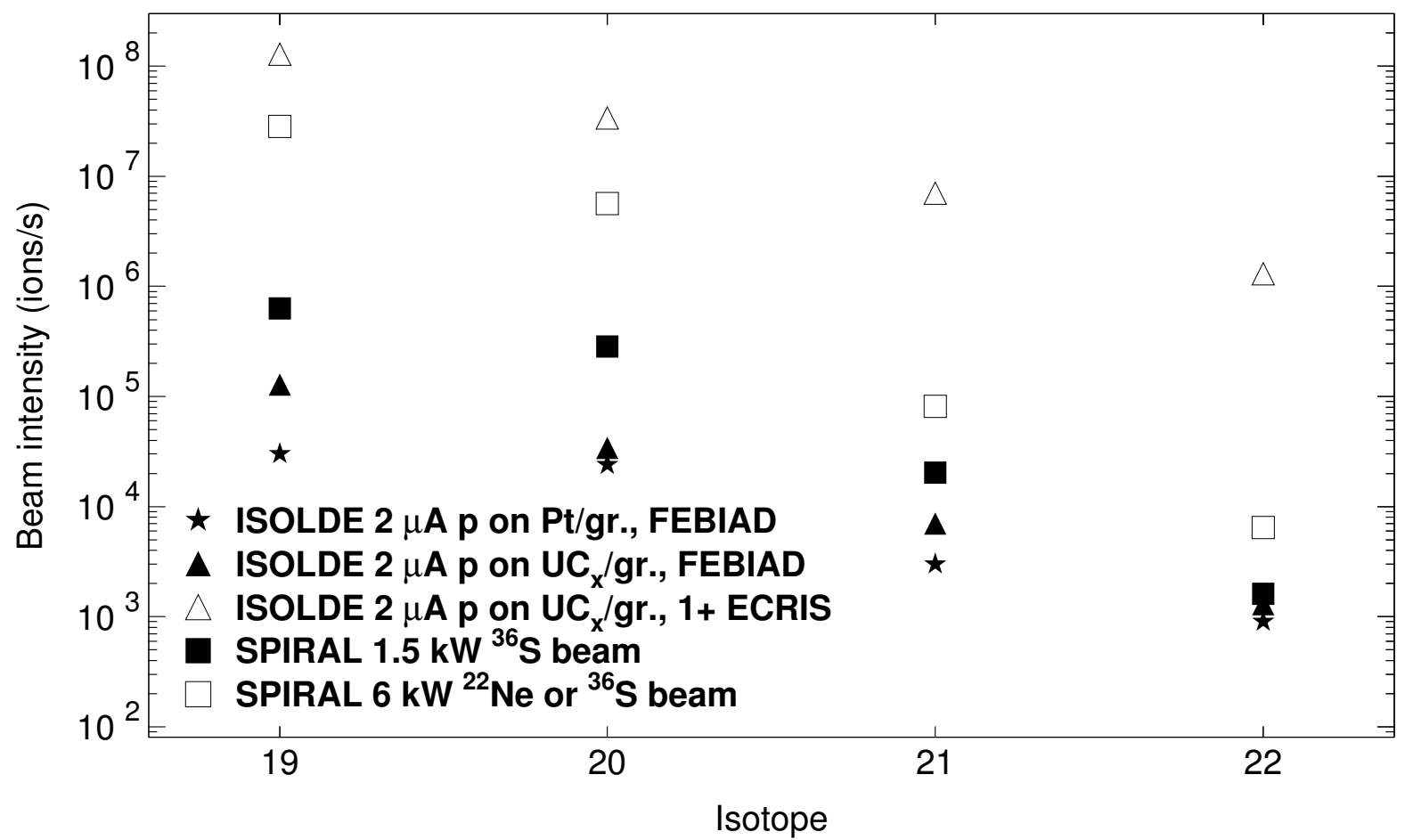

Figure 2: Measured (full symbols) and extrapolated (open symbols) beam intensities for neutron-rich oxygen isotopes. 